\title{
Fatores predisponentes na prevalência da candidíase vulvovaginal
}

\author{
Factors predicting in the prevalence of vulvovaginal candidiasis
}

\author{
Haryne Lizandrey Azevedo Furtado ${ }^{1}$, Brenda Letícia Araujo Motta ${ }^{2}$, Thayariane Lira \\ Mendes $^{3}$, Thayomara Oliveira da Silva ${ }^{4}$, Julliana Ribeiro Alves dos Santos ${ }^{5}$.
}

RESUMO: A Candidíase Vulvovaginal (CVV) é um dos principais problemas ginecológicos. Sua prevalência parece ter aumentado nos últimos anos, evoluindo de 0,5\% em 1968 para 22,5\% em 1998, época em que se tornou a causa mais comum de infecção da mucosa vulvovaginal. Aproximadamente $5 \%$ das mulheres diagnosticadas desenvolvem candidíase vulvovaginal recorrente (CVVR). A CVV possui vários fatores de risco e é resistente a maioria dos antifúngicos. O objetivo deste estudo é analisar a prevalência de candidíase vulvovaginal e os seus fatores de riscos, abrangendo diagnóstico e tratamento por meio de revisão bibliográfica, análise realizada no período de Janeiro a Julho de 2018, utilizando os descritores: Candidíase Vulvovaginal, Prevalência e Fatores de Risco, sendo utilizados 21 dos 26 artigos encontrados. As espécies mais predominantes na CVV dependem do local de estudo e os principais fatores predisponentes são a falta de conhecimento sobre a candidíase, fatores geográficos, hábitos culturais, entre outros. Existe uma falta de concordância entre os autores em relação aos fatores de risco, sendo necessárias novas pesquisas para propor medidas preventivas para a candidíase vulvovaginal. PALAVRAS-CHAVE: Candidíase Vulvovaginal; Prevalência; Fatores de Risco.

ABSTRACT: Vulvovaginal Candidiasis (CVV) is one of the major gynecological problems. Its prevalence appears to have increased in recent years, rising from $0.5 \%$ in 1968 to $22.5 \%$ in 1998 , when it became the most common cause of vulvovaginal mucosal infection. Approximately $5 \%$ of women diagnosed develop recurrent vulvovaginal candidiasis (CVVR). CVV has several risk factors and it is resistant to most antifungal agents. The objective of this study is to analyze the prevalence of vulvovaginal candidiasis and its risk factors, including diagnosis and treatment through a bibliographical review, performed in the period from January to July 2018, using the descriptors: Vulvovaginal Candidiasis, Prevalence and Risk, using 21 of the 26 articles found. The most prevalent species in CVV depend on the place of study and the main predisposing factors are lack of knowledge about candidiasis, geographic factors, cultural habits, among others. There is a lack of agreement between the authors regarding the risk factors, and new research is needed to propose preventive measures for vulvovaginal candidiasis.

KEYWORDS: Vulvovaginal Candidiasis; Prevalence; Risk factors.

${ }^{1}$ Discente do $7^{\circ}$ período curso de Biomedicina e do Laboratório de Microbiologia Ambiental (LAMAM) da Universidade CEUMA, Campus Renascença, E-mail: haryne.lizandrey@gmail.com ${ }^{2}$ Graduanda em Biomedicina e do Laboratório de Microbiologia Ambiental (LAMAM) da Universidade CEUMA, Campus Renascença, E-mail: brendaaraujo_biomed@hotmail.com ${ }^{3}$ Discente do $7^{\circ}$ período curso de Biomedicina e do Laboratório de Microbiologia Ambiental (LAMAM) da Universidade CEUMA, Campus Renascença, E-mail: thayariane@yahoo.com ${ }^{4}$ Discente do $5^{\circ}$ período curso de Biomedicina e do Laboratório de Microbiologia Ambiental (LAMAM) da Universidade CEUMA, Campus Renascença, E-mail: thayomara10oliveira@gmail.com

${ }^{5}$ Docente do curso de Biomedicina e do Mestrado em Meio Ambiente e do Mestrado em Biologia Microbiana; Laboratório de Microbiologia Ambiental (LAMAM) da Universidade CEUMA, Campus Renascença, E-mail: julliana.santos85@gmail.com

Autor correspondente: Prof. ․ Dra. Julliana Ribeiro Alves dos Santos, (Rua Josué Montello, no 01, Renascença II, CEP: 65075-120), São Luís, MA, Brasil, Tel.: (98) 982652044.

\section{Introdução}

Os fungos são organismos
eucarióticos unicelulares ou
pluricelulares,


apresentar uma forma arredondada, filamentosa, ou então, uma combinação de ambas as formas. Alguns fungos fazem parte da microbiota comensal humana, obtendo benefícios sem causar prejuízos ao hospedeiro. São tipicamente encontrados na mucosa oral, vaginal e gastrointestinal ou residentes na pele e no epitélio respiratório. Quando ocorrem alterações homeostáticas em um indivíduo, alguns organismos comensais podem passar a ser patogênicos ${ }^{1,2}$.

Um exemplo a ser citado é Candida spp., pertencente ao filo Ascomycota. São leveduras comensais das mucosas vaginal e digestiva, se tornam patogênicas durante determinadas condições que alteram 0 ambiente vaginal, favorecendo a colonização das leveduras, ocasionando a Candidíase Vulvovaginal (CVV), conceituada como uma infecção da vulva e da vagina, com ocorrências de prurido vulvar intenso, leucorréia, dispareunia, disúria, edema e eritema vulvovaginal, sendo prurido o sintoma mais importante quando a CVV é comparada a vulvovaginites de outra etiologia ${ }^{3,4}$. Incluem-se neste espectro, pacientes com ou sem sintomas cujo diagnóstico foi estabelecido por cultura positiva de secreção vaginal ${ }^{5}$.

As manifestações clínicas na candidíase apresentam grande diversidade de quadros, como candidíase cutânea mucosa e candidíase invasiva ou sistêmica ${ }^{6}$. As leveduras são carreadas para a vagina por meio de processo de auto transmissão a partir da região perianal, tendo como fonte a microbiota do próprio intestino ou a troca com o parceiro por via sexual.
Entretanto, estes microrganismos costumam permanecer na mucosa vaginal apenas como colonizantes e, uma vez encontrando condições apropriadas, aceleram o processo de multiplicação e expressam fatores de virulência, culminado com a invasão da mucosa e ocasionando a CVV sintomática ${ }^{7}$.

Algumas condições são consideradas fatores de risco para vulvovaginites, como gravidez, o uso de contraceptivos orais, higiene, predisposição genética, antibioticoterapia prévia, doenças imunossupressoras como o HIV, Diabetes Mellitus (DM) devido aos níveis mais altos de glicogênio. Ocorre principalmente em mulheres adultas e em idade fértil, apresentam lesões brancas, cremosas e planas. Aproximadamente $\quad 5-8 \%$ das mulheres desenvolvem a Candidíase Vulvovaginal Recorrente (CVVR), a qual apesar de representar um problema de importância global na saúde pública, a sua incidência exata é desconhecida ${ }^{8,9}$.

Diante do exposto, o estudo objetivou realizar um levantamento bibliográfico, com a finalidade de analisar os fatores de riscos e a prevalência de candidíase vulvovaginal no Brasil, abrangendo o diagnóstico e 0 tratamento da infecção.

\section{Material e método}

O presente artigo trata de um estudo de revisão bibliográfica, utilizando artigos já publicados disponíveis nas bibliotecas virtuais Scielo, Google acadêmico e Lilacs, entre os anos de 1999 a 2017, usando os descritores: candidíase 
vulvovaginal; prevalência; fatores de risco. A pesquisa foi realizada de Janeiro a Junho de 2018, sendo estudados 26 artigos, destes 5 foram excluídos, pois não atendiam ao critério de escolha, sendo este os artigos que continham como conteúdo os fatores de risco da patologia, definição e as espécies de Candida mais prevalentes, com os dados de diferentes estados do Brasil.

\section{Resultados e discussões}

\section{Epidemiologia e Fatores de Risco}

A CVV está entre os principais problemas ginecológicos. Sua prevalência parece ter aumentado nos últimos anos evoluindo de $0,5 \%$ em 1968 para 22,5\% em 1998, época em que se tornou a causa mais comum de infecção vaginal ${ }^{7}$. É considerada a segunda causa mais comum de infecção genital em mulheres em idade reprodutiva, cuja taxa de recorrência é de $40-50 \%$, e aproximadamente 5-8\% desenvolvem CVVR, que é caracterizada por quatro ou mais episódios da doença ao longo de um período de doze meses ${ }^{8}$.

Um estudo conduzido entre julho a agosto de 2012 em uma comunidade ribeirinha do Município de Imperatriz, no Estado do Maranhão, com residentes à margem do rio Tocantins, mostrou que o perfil sociodemográfico que apresentou a presença do fungo prevaleceu na faixa etária de 20 a 39 anos $(51,4 \%)$, em mulheres de cor parda $(66,4 \%)$, casadas $(30,8 \%)$, com menor grau de escolaridade, com renda de até três salários mínimos (58\%) e em domésticas do $\operatorname{lar}(63,6 \%)^{10}$.

Numerosos estudos indicam que C. albicans (80-90\%) é a levedura mais frequente nos casos de CVV. Entretanto, nos últimos anos, tem-se observado um aumento de 10 a $20 \%$ na frequência das espécies não $C$. albicans, principalmente $C$. glabrata, $C$. tropicalis, C. krusei, C. parapsilosis, C. pseudotropicalis, C.lusitaniae e $C$. guillermondi, indicando uma tendência de mudança na etiologia da candidíase, após décadas de predomínio de C. albicans ${ }^{3,11,12}$. A maior preocupação reside no fato de que essas outras espécies, em geral, tendem a ser mais resistentes aos antifúngicos ${ }^{7}$.

\begin{tabular}{lll}
\multicolumn{2}{c}{ Publicações } & sobre a \\
prevalência de candidíase & de \\
vulvovaginal, com & diagnóstico
\end{tabular} definido por cultura, ainda são pouco comuns, sendo que alguns estudos se baseiam apenas em autodiagnóstico ou em diagnóstico clínico. Estudo desenvolvido na Universidade de Michigan (EUA) estimou que. Provavelmente. 55,7\% de todas as mulheres terão pelo menos um episódio de vulvovaginites por Candida spp. ao longo de suas vidas. No Brasil, os dados epidemiológicos são bem mais escassos ${ }^{5}$.

Estudo transversal realizado em 1996, incluindo 72 mulheres não grávidas que procuraram o Serviço de Planejamento Familiar do Hospital das Clínicas da Universidade Federal de Minas Gerais, observou uma prevalência de candidíase vulvovaginal de $25 \%$, confirmado por cultura ${ }^{13}$. Outro estudo transversal realizado em 1998-1999, que avaliou 205 mulheres atendidas no ambulatório 
de Ginecologia e Obstetrícia da Universidade do Espírito Santo, demonstrou prevalência de $25 \%$ de candidíase vulvovaginal entre as mulheres assintomáticas e de $60 \%$ entre as que apresentavam sintomas de vulvovaginites ${ }^{14}$.

Um estudo produzido entre agosto de 2004 e junho de 2005, em Frederico Westphalen, Rio Grande do Sul, com 702 pacientes atendidos em três unidades de saúde, dos quais $43(5,8 \%)$ foram positivos para candidíase vulvovaginal, destes, 24 foram selecionados para a pesquisa, $50 \%$ das pacientes possuíam de 21 a 30 anos; e 14 pacientes (58,3\%) eram reincidentes, sendo 3 diabéticas; 10 pacientes $(41,7 \%)$ apresentaram a infecção durante o verão. De acordo com os testes fenotípicos, 11 (45,8\%) foram identificadas como Candida albicans, 9 (37,5\%) como Candida tropicalis e $4(16,7 \%)$ como Candida glabrata ${ }^{15}$.

Em outro estudo feito na Baixada Fluminense, Rio de Janeiro, foram identificadas três espécies de fungos do gênero Candida entre as 73 mulheres amostradas, onde $C$. albicans teve coeficiente de prevalência (CP) de 89,04\%; para $C$. krusei a prevalência calculada foi $\mathrm{CP}$ $=4,11 \%$; e para $C$. tropicalis $\mathrm{CP}=$ $6,85 \%$. Os autores comentaram ainda que a idade é um fator importante nesse tipo de processo patológico e que, durante os anos de ciclo menstrual, espécies do gênero Candida figuram entre os agentes microbianos causadores de vulvovaginites $^{16}$.

Já em São Luís do Maranhão, um estudo realizado com 144 pacientes no período de outubro de 2010 a maio de 2011 mostrou que destas, 90 mulheres $(62,5 \%)$ tiverem culturas positivas para leveduras do gênero Candida (Figura 1), demonstrando maior prevalência das espécies $C$. parapsilosis $(43,3 \%)$, C. glabrata $(16,7 \%)$ e $C$. albicans $(15,6 \%)$. As demais espécies, C. tropicalis, C. famata, $C$. krusei, $C$. sake e C. globosa apresentaram frequência isolada inferior a $10 \%$ dos casos $^{17}$.

Figura 1. Frequência relativa das espécies mais prevalentes de leveduras isoladas de seçreção vaginal de pacientes atendidas no Hospital da Mulher em 2013. São Luís, MA.

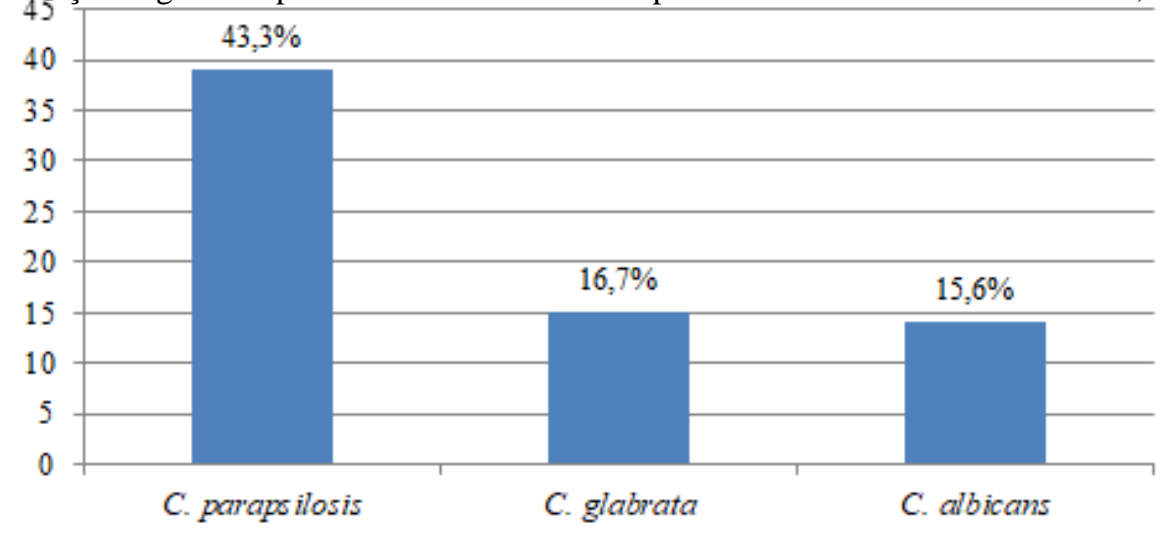

Fonte: Adaptado de Alves et al., 2015.

Fatores de Risco

Existem variações geográficas significativas no padrão 
da Candida spp. cepas isoladas. Embora a América do Norte tenha uma prevalência de $C$. glabrata, na América do Sul há uma predominância de $C$. parapsilosis e $\quad$. tropicalis entre espécies de Candida não-albicans ${ }^{18}$.

Em estudo realizado com 286 mulheres dos Municípios de llhéus e Itabuna no Estado da Bahia, foi observado que a posição geográfica também pode ser considerada um fator relevante para o aparecimento da infecção, assim como o uso frequente de calça jeans, procedimentos invasivos e imunossupressão. Portanto, essa prevalência parece variar de acordo com o meio diagnóstico utilizado, com as características das populações estudadas e outros fatores interferentes no diagnóstico ${ }^{10}$.

As diferenças quanto ao isolamento de Candida spp. a partir da mucosa vaginal podem ser explicadas por hábitos culturais de diferentes regiões, especialmente no que se refere às práticas de higiene, pois este fator está diretamente relacionado à autocontaminação porque a levedura pertence à microbiota normal do trato gastrointestinal. Além disso, estudos em diferentes regiões do Brasil sugerem que fatores geográficos, tais como condições climáticas, e fatores sociais e ambientais interferem na prevalência de espécies de Candida, bem como na sensibilidade dos isolados aos antifúngicos ${ }^{8}$.

A "falta de conhecimento" sobre candidíase foi considerada como fator predisponente obtendo $77 \%$ de positividade para Candida, diferente do fumo que apresentou apenas 10,9\%; o uso de contraceptivos orais $(19,8 \%)$; uso de preservativos sexuais (27,5\%); uso de medicamentos como antibiótico e corticosteroides, juntos, contribuem para 76,9\%; enquanto câncer e hipertensão arterial não mostraram diferença significativa quando avaliados como fatores predisponentes para a infecção ${ }^{10}$.

\section{Candidíase Recorrente (CVVR): \\ Vulvovaginal}

Aproximadamente $5 \%$ das mulheres com CVV desenvolvem Candidíase Vulvovaginal Recorrente (CVVR), definida usualmente como a ocorrência de quatro ou mais episódios de CVV no período de 12 meses. Ao contrário das mulheres que têm episódios esporádicos de CVV, aquelas com a doença recorrente não se beneficiam de uma diminuição na frequência de episódios sintomáticos com o passar da idade. A patogênese da CVVR entre mulheres que não têm condições predisponentes aparentes, que são a grande maioria, ainda permanece sob investigação. Uma elevação na resistência de espécies de Candida não tem sido observada na maioria dos casos, embora se analisarmos essas pacientes como um grupo, mulheres com recorrência têm uma prevalência discretamente mais elevada de $C$. glabrata, menos sensível às drogas imidazólicas, comumente utilizadas no tratamento de CVV e CVVR ${ }^{11}$.

\section{Diagnóstico}

O exame ginecológico evidenciará o edema, o corrimento característico, as fissuras e a 
hiperemia das paredes vaginais. Porém, para a comprovação do diagnóstico de candidíase, deve ser realizado um exame específico, como a microscopia onde deverão estar presentes hifas e esporos; o imunodiagnóstico, que consiste na pesquisa de anticorpos e antígenos fúngicos; ou culturas em meios como Agar Sabouraud. O diagnóstico por cultura é mais especifico, sendo possível dar resultado negativo na microscopia e positivo na cultura ${ }^{19}$.

Algumas técnicas para a identificação das espécies de Candida são empregadas, dentre as quais, é possível incluir: a de fermentação de açúcares; a análise morfológica da colônia (prova do microcultivo); e testes que utilizam agentes cromogênicos e enzimáticos como 0 meio ágar seletivo e diferencial CHROMagar Candida, que faz uso de substrato $\beta$ glicosaminidase e diferencia as leveduras baseado na cor e aspecto das colônias, a mudança de coloração se dá pela degradação de compostos cromogênicos por enzimas específicas produzidas pelos fungos. As colônias de coloração verde são classificadas como pertencentes à $C$. albicans, colônias com coloração azul a $C$. tropicalis, colônias rosa e rugosa a C. krusei, as de coloração lilás são sugestivas de $C$. glabrata e outras espécies de Candida (Candida spp.) apresentam coloração branca à violeta $^{10}$.

\section{Tratamento}

Dentre os antifúngicos utilizados na terapêutica de candidíases invasivas destacam-se triazólicos, como fluconazol, derivados poliênicos, como anfotericina $B$, e, do grupo das equinocandinas, a caspofungina ${ }^{6}$. Os agentes poliméricos são outro grupo de drogas utilizadas para o tratamento de CVV; Este grupo inclui a nistatina, que é a mais utilizada no Brasil e está livremente disponível no Sistema Único de Saúde (SUS). O conhecimento dos padrões de susceptibilidade a fármacos típicos em isolados de diferentes regiões do Brasil não é rotineiramente realizado, mas este conhecimento permitirá a racionalização do uso empírico de agentes antifúngicos, contribuindo assim para o controle da disseminação de resistência a drogas $^{8}$.

A resistência aos antifúngicos, clínica ou in vitro, pode ocorrer quando células de fungos susceptíveis passam a serem resistentes devido ao contato prévio com a droga ou é inerente ao próprio microrganismo. Esta resistência ocorre quando as alterações na rota da biossíntese dos esteróis e da expressão do gene ERG 11 envolvidos na síntese da enzima 14 a-Demetilase reduzem o acúmulo intracelular da droga e a inativação da mesma ${ }^{20}$. Pode ser resultado de terapia em pacientes imunodeprimidos, neutropênicos, com infecção em tecidos pouco vascularizados ou abcessos fechados, falta de aderência do paciente ao tratamento e formação de biofilmes em próteses e cateteres. Várias espécies de leveduras do gênero Candida têm a habilidade de produzir biofilme, inclusive $C$. albicans ${ }^{6}$.

Devido aos riscos associados ao uso de medicamentos e ao crescente índice de casos de recorrência, nota-se uma lacuna 
científica em relação a novas possibilidades terapêuticas viáveis. Terapias não medicamentosas vêm sendo desenvolvidas no tratamento desta patologia, já que são poucos os recursos disponíveis para tratar casos de resistência fúngica ${ }^{21}$.

\section{Conclusão}

Os artigos sobre a CVV e a CVVR destacaram um fator comum para o aparecimento e recorrência da CVV que foram a posição geográfica; o clima; o uso frequente de calça jeans e o uso indiscriminado de antifúngicos, levando à resistência das espécies às drogas de uso clínico. Todos os relatos evidenciam um aumento na prevalência da patologia nos últimos anos, e notou-se uma falta de estudos e poucas publicações contendo a incidência e prevalência de casos de CVV e CVVR no Brasil. Existe uma falta de concordância entre a maioria dos fatores de risco, sendo necessária uma pesquisa mais aprofundada acerca da CVV e da CVVR para que medidas preventivas possam ser adequadamente estabelecidas.

\section{Referências}

1. Neves, R. das. Educação. Biologia. Microbiologia. Fungos. Disponível em <educacao.globo.com/biologia/assunto/ microbiologia/fungos.html>. Acesso em 29 de Junho de 2018.
2. Plas, R. V. D. Candidíase oral: Manifestações clínicas e Tratamento. [dissertação]. Porto: Faculdade de Ciências da Saúde, Universidade Fernando Pessoa, 2016.

3. Holanda, A. A. R.; Fernandes, A. C. S.; Bezerra, C. M.; Ferreira, M. A. F.; Holanda, M. R. R.; Holanda, J. C. P.; Milan, E. P. Candidíase vulvovaginal: sintomatologia, fatores de risco e colonização anal concomitante. Rev. Bras. Ginecol. Obstet. 2007 Jan; 29 (1): 3-9.

4. Gunther, L. S. A.; Martins, H. P. R.; Gimenes, F.; Abreu, A. L. P.; Consolaro, M. E. L.; Svidzinski, T. I. E. Prevalence of Candida albicans and non-albicans isolates from vaginal secretions: comparative evaluation of colonization, vaginal candidiasis and recurrent vaginal candidiasis in diabetic and non-diabetic women. São Paulo Med. J. 2014; 132 (2): 116-120.

5. Rosa, M. I. Da; Rumel, D. Fatores associados à candidíase vulvovaginal: estudo exploratório. Rev. Bras. Ginecol. Obstet. 2004 Fev; 26 (1): 5-70.

6. Vieira, A. J. H.; Santos, J. I. Mecanismos de resistência de Candida albicans aos antifúngicos anfotericina $B$, fluconazol e caspofungina. RBAC. 2017; 49 (3): 2359.

7. Ferrazza, M. H. S. H.; Maluf, M. L. F.; Consolaro, M. E. L.; Shinobu, C. S.; Svidzinski, T. I. E.; Batista, M. R. Caracterização de leveduras isoladas da vagina e sua associação com candidíase vulvovaginal em duas cidades do sul do Brasil. Rev. Bras. Ginecol. Obstet. 2005 Fev; 27 (2): 58-63.

8. Brandolt, T. M.; Klafke, G. B.; Gonçalves, C. V.; Bitencourt, L. R.; Martinez, A. M. B. De; Mendes, J. F.; Meireles, M. C. A.; Xavier, M. O. Prevalence of Candida spp. in cervical-vaginal samples and the in vitro susceptibility of isolates. Braz. J. Microbiol. 2017 Mar; 48 (1): 145-150.

9. Tozzo, A. B.; Grazziotin, N. A. Candidíase vulvovaginal. Perspectiva. 2012 Mar; 36 (133): 53-62.

10. Sá, M. C. N.; Sousa, H. R.; Amaro, C. S. O.; Pinheiro, D. N.; Oliveira, M. M. M.; Pinheiro, M.C. N. Isolamento de Candida no esfregaço cérvico-vaginal de 
mulheres não gestantes residentes em área ribeirinha do Estado do Maranhão, Brasil, 2012. RevPan-AmazSaude. 2014; 5 (1): 25-34.

11. Alvares, C. A.; Svidzinski, T. I. E.; Consolaro, M. E. L. Candidíase vulvovaginal: fatores predisponentes do hospedeiro e virulência das leveduras. J. Bras. Patol. Med. Lab. 2007 Out; 43 (5): 319-327.

12. Colombo, A. L.; Guimaraes, T. Epidemiologia das infecções hematogênicas por Candida spp. Rev. Soc. Bras. Med. Trop. 2003 Out; 36 (5): 599-607.

13. Aleixo Neto, A.; Hamdan, J. S.; Souza, R. C. Prevalência de cândida na flora vaginal de mulheres atendidas num serviço de planejamento familiar. Rev. Bras. Ginecol. Obstet. 1999; 21 (8): 441 445.

14. Ribeiro, M. A.; Dietze, R.; Paula, C. R.; Da Mata, D. A.; Colombo, A. L. Susceptibility profile of vaginal yeast isolates from Brazil. Mycopathologia. 2001; 151: 5-10.

15. Linares, C. E. B.; Dagios, G.; Carati, M. R.; Gasparin, M. P.; Neto, A. T.; Scheid, L. A.; Schubert, A. Estudo Epidemiológico e Perfil da Suscetibilidade de Amostras de Candida Isoladas de Mulheres dom Candidiase Vulvovaginal em Frederico Westphalen RS. Saúde. 2005; 31 (1-2): 42 - 46.

16. Norberg, A. N.; Helena, A. A. S.; Madeira-Oliveira, J. T.; Sanches, F. G.; Ribeiro, P. C.; Machado, A. N.; Freire, N. M. S. Prevalência de Candidíase Vulvovaginal em Mulheres da Região da Baixada Fluminense, Estado do Rio De Janeiro, Brasil. Revista da Faculdade de Ciências Gerenciais de Manhuaçu. 2015 Jan-Jun; 12 (1): 109-114.

17. Alves, M. B.; Silva, Í. M. De O.; Santos, C. I. Dos; França, Y. R.; Oliveira, S. K. R.; Monteiro, S. G.; Monteiro, C. de A. Prevalência de Candida spp. em amostras de secreção vaginal e sua relação com fatores associados à vulvovaginite. Rev. Investig. Bioméd. 2015; 7 (1): 58-68.

18. Khouri, S.; Ruiz, L. S.; Auler, M. E.; Silva, B. C. M.; Pereira, V. B. R.; Domaneschi, C.; Hahn, R. C.; Paula, C. R. Evaluation of infections by Candida at a university hospital of Vale do Paraíba region, São Paulo State, Brazil: species distribution, colonization, risk factors and antifungal susceptibility. Rev Pan-Amaz Saude. 2016; 7 (2): 51-57.

19. Freitas, B. de; Pires, D. V. D. da C. P. Fatores de Risco Associados à Candidíase Vulvovaginal. Revista Eletrônica Saúde em Foco. 2016; 8: 247252.

20. Zardo, V.; Mezzari, A. Os antifúngicos nas infecções por Candida sp. NewsLab. 2004; 63: 136-146.

21. Leal, M. R. D.; Lima, M. C. N. P. C.; Klein, S. de O. T.; Lordêlo, P. Tratamento Da Candidíase Vulvovaginal e Novas Perspectivas Terapêuticas: Uma Revisão Narrativa. Revista Pesquisa em Fisioterapia. 2016 Nov; 6 (4): 462-467. 